\title{
Limis for Production or Consumption of Energy and Substances Predicted by Thermodynamics
}

\author{
S. Sieniutycz \\ Faculty of Chemical and Process Engineering, Warsaw University of Technology, \\ 1 Warynskiego Street, 00-645 Warsaw (Poland) \\ Phone 00-48-22-256340; fax: 00-48-22-251440, e-mail sieniutycz@ichip.pw.edu.pl
}

\begin{abstract}
We describe quantitatively effects of nonlinear transfer phenomena that drive energy generators (thermal engines) and heat pumps. it is shown that these transports can be treated either in a standard way or as peculiar chemical reactions described by appropriate affinities. An approach to nonlinear transports links heat fluxes with differences of temperature in certain power $t^{\mathrm{a}}$. a more recent approach distinguishes in each elementary transfer step two competitive (unidirectional) fluxes and the resulting flux as their difference. we show how the kinetics of this sort can be implemented into the contemporary theory of thermal energy generators.
\end{abstract}

\section{Key words}

Energy limits, exergy, power yield, engines, exergy, second law.

\section{Introduction}

In this work basic rules in modeling the energy limits and efficiency drop caused by finite rates of real processes in the system are discussed. In particular, radiation engines are analyzed as important systems governed by nonlinear laws of thermodynamics and transport. A general formula linking a converter's efficiency with the cumulative power and entropy production is applied to estimate an irreversible limit for power yield and to define a finite-rate extension of the classical work potential. The real work is the cumulative effect obtained from a resource fluid at flow, a set of sequentially arranged engines, and an infinite bath. The use of optimal control methods, such as maximum principles and dynamic programming, leads to a finite-rate generalization of the classical available energy.

In this research, the energy limits for imperfect cycles producing or consuming mechanical energy are investigated in the framework of finite time thermodynamics by taking the maximum power as the objective. Since the heat transfer law has a strong effect on the performance of the cycle an approach is pursued that aspires to derive a relatively large number of basic equations in the form independent on the mode of energy transfer between the circulating fluid and reservoirs. In the nonlinear modeling that is methodological extension of that known for the linear case the heat transfer is assumed to obey the power law $T^{\mathrm{a}}$ (heat flux proportional to the difference in $T^{\mathrm{a}}$ ) instead of Newton's linear law. A more recent approach is also briefly considered in which one distinguishes in each elementary transfer process two competitive (unidirectional) fluxes and the resulting flux being their difference. The unidirectional fluxes are equal in the thermodynamic equilibrium and their difference off the equilibrium constitutes the observed flux representing the resulting rate of the process. In this approach nonlinear resistance and of a related affinity play important role. In most general situation when the mass transfer is included the nonequilibrium transports are described by equations containing exponential terms with respect to chemical potentials of Planck and temperature reciprocal, that simultaneously are analytical expressions characterizing the transport of the substance or energy by the energy barrier. We show how the kinetics of this sort can be implemented into the contemporary theory of thermal energy generators.

\section{Problem formulation}

The classical exergy defines bounds on the common work delivered from (or supplied to) slow, reversible processes [1]. Such bounds are reversible as the magnitude of the work delivered during the reversible approach to equilibrium is equal to the one of the work supplied, after the initial and final states are inverted, i.e. when the second process reverses to the initial state of the first. Our research is towards generalization of the classical exergy for finite rates. During the approach to the equilibrium the so-called engine mode of the system takes place in which the work is released, during the departure- the so-called heat-pump mode occurs in which work is supplied. Work $\mathrm{W}$ delivered in the engine mode is positive by assumption. In the heat-pump mode $\mathrm{W}$ is negative, or the positive work (-W) must be supplied to the system. To find a generalized exergy, optimization problems are set, for the maximum of the work delivered [max $\mathrm{W}]$ and for the minimum of the work supplied $[\min (-\mathrm{W})]$. We show that while the reversibility property is lost for such exergy, its (kinetic) bounds are stronger and more useful than classical thermostatic bounds. This substantiates role of the extended exergy for evaluation of energy limits in practical systems.

With functionals of power generation (consumption) at disposal we can formulate the Hamilton-Jacobi-Bellman 
theory (HJB theory) for the extended exergy and related extremum work. The HJB theory is the basic ingredient in variational calculus and optimal control [2-4]. A HJB equation extends the classical Hamilton-Jacobi equation [5] by addition of extremum conditions, and it is essential to develop numerical methods in complex cases (with state dependent coefficients) when the HJB equation of the problem cannot be solved analytically. Due to the direct link between the HJB theory and dynamic programming the associated numerical methods make use the Bellman's recurrence equation. These methods are complementary with respect of the Pontryagin's principle [6], as both are effective seeking methods of functional extrema. Yet, in spite of its power, Pontriagin's principle does not yield the principal function $\mathrm{V}$ which is in our case a general work potential describing the change of the extended exergy, the main result being sought. Otherwise, when a HJB equation is known, the exergy (or work) is explicit, and the discrete numerical problem leads to Bellman's recurrence equation, solvable by dynamic programming [7]. Our problem of generalized exergy falls into the category of finite-time potentials, an important problem of contemporary thermodynamics [8]. In this paper we solve the problem of extremum work by using the concept of multistage energy production or consumption, where each stage is the so-called Curzon-Ahlborn-Novikov process $[8,9]$. The concept of single irreversible stage is illustrated in Fig. 1 that presents the temperature-entropy diagram of an arbitrary irreversible stage. Each stage can work either in the heat-pump mode (larger, external loop in Fig. 1) or in the engine mode (smaller, internal loop in Fig. 1).

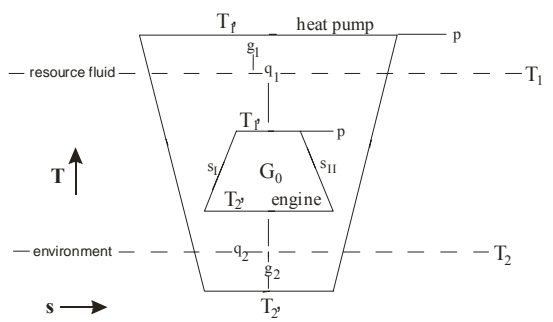

Fig.1 Two basic modes with internal and external dissipation: power yield in an engine and power consumption in a heat pump. Primed temperatures characterize the circulating fluid.

Our analysis here extends the previous analyses of the problem [8-11] by taking into account internal irreversibilities within the thermal machines at each stage of the operation following the recent method that applies the factor of internal irreversibilities, $\Phi$ [12]. By definition, $\Phi=\Delta \mathrm{S}_{2}, / \Delta \mathrm{S}_{1}$, (where $\Delta \mathrm{S}_{1}$, and $\Delta \mathrm{S}_{2}$, are respectively the entropy changes of the circulating fluid along the two isotherms $T_{1}$, and $T_{2}$, in Figure 1) equals the ratio of the entropy fluxes across the thermal machine, $\Phi=J_{\mathrm{s} 2} / J_{\mathrm{s} 1}$. Due to the second law inequality at the steady state the following inequalities are valid: $J_{\mathrm{s} 2} / J_{\mathrm{s} 1}>1$ for engines and $J_{\mathrm{s} 2}, J_{\mathrm{s} 1}<1$ for heat pumps; thus the considered ratio $\Phi$ measures the process irreversibility. In fact, $\Phi$ is a synthetic measure of the machine's imperfection. $\Phi$ satisfies inequality $\Phi>1$ for engine mode and $\Phi<1$ for heat pump mode of the system. Our purpose is to derive a generalized exergy in terms of $\Phi$.

\section{Entropy Production and Process Efficiency}

We shall present here a shortest possible proof of the formula describing the real work by using the so-called Gouy-Stodola law that links the lost work with the entropy production [1]. In the analysis we shall make use of the fact that the thermal efficiency of any real thermal engine can always by written in the form $\eta=1-d Q_{2} / d Q_{1}$. By evaluating total entropy production at the stage (the sum of external and internal parts) $\mathrm{S}_{\sigma}$ as the difference between the outlet and inlet entropy fluxes we find in terms of the first-law efficiency $\eta$

$$
d S_{\sigma}=\frac{d Q_{1}(1-\eta)}{T_{2}}-\frac{d Q_{1}}{T_{1}}=\frac{d Q_{1}}{T_{2}}\left(1-\eta-T_{2} / T_{1}\right)
$$

This is a general equation as there was not any special assumptions involved in its derivation. It states that the entropy production in an arbitrary thermal engine is directly related to the deviation of the engine's efficiency from the corresponding Carnot efficiency. This conclusion leads us to an important analytical formula for the total entropy source that will enable its direct optimization. The entropy balance of the thermal machine contains the internal entropy production as the source term in the expression

$$
\frac{d Q_{2}}{T_{2^{\prime}}}-\frac{d Q_{1}}{T_{1^{\prime}}}=d S_{\sigma}^{\text {int }}
$$

In terms of the coefficient $\Phi=1+T_{1} d S_{\sigma}^{\text {int }} / d Q_{1}$ called the internal irreversibility factor the entropy balance of the internal part of the system takes the form usually applied for thermal machines

$$
\Phi \frac{d Q_{1}}{T_{1^{\prime}}}=\frac{d Q_{2}}{T_{2^{\prime}}}
$$

One can evaluate $\Phi$ from the average value of the internal entropy production that describes the effect of irreversible processes within the thermal machine. Clearly, in many cases $\Phi$ is a complicated function of the machine's operating variables. In those complex cases one applies the data of $\sigma_{s}^{\text {int }}=d S_{\sigma}^{\text {int }} / d t$ to calculate an averaged value of the coefficient $\Phi$. In the analysis of the operation considered the quantity $\Phi$ is treated as the process constant. In other words, it is an average value of $\Phi$, evaluated within the boundaries of operative parameters of interest that is used in most of the analyses of thermal machines including the present one. For chillers and energy generators experimental data of $\sigma_{s}^{\text {int }}=d S_{\sigma}^{\text {int }} / d t$ are available that allow the calculation of $\Phi$; see ref. [12] for more information.

Consequently, the efficiency $\eta$ can be evaluated in terms of the parameters characterizing the thermal machine

$$
\eta=1-\frac{d Q_{2}}{d Q_{1}}=1-\Phi \frac{T_{2^{\prime}}}{T_{1^{\prime}}}=1-\left(1+T_{1^{\prime}} \frac{d S_{\sigma}^{\mathrm{int}}}{d Q_{1}}\right) \frac{T_{2^{\prime}}}{T_{1^{\prime}}}
$$

After eliminating $\eta$ from eqs. (1) and (4) we conclude that, quite generally, the total entropy production can be written in a transformed form 


$$
d S_{\sigma}=\frac{d Q_{1}}{T_{2}}\left(\Phi \frac{T_{2^{\prime}}}{T_{1^{\prime}}}-\frac{T_{2}}{T_{1}}\right)=d Q_{1}\left(\frac{(\Phi-1)}{T^{\prime}}+\left(\frac{1}{T^{\prime}}-\frac{1}{T_{1}}\right)\right)
$$

The first term in the resulting expression of eq. (5) describes the internal entropy source (within the thermal machine) and the second one the external entropy source (within the reservoirs). Equivalently, after using the definition of the internal irreversibility factor $\Phi=1+T_{1} d S_{\sigma}^{\text {int }} / d Q_{1}$

$$
d S_{\sigma}=\frac{T_{1^{\prime}}}{T^{\prime}} d S_{\sigma}^{\mathrm{int}}+d Q_{1}\left(\frac{1}{T^{\prime}}-\frac{1}{T_{1}}\right)
$$

In the last two equations the structural quantity called the Carnot temperature $T$, was introduced that satisfies the thermodynamic relation

$$
T^{\prime} \equiv T_{2} T_{1^{\prime}} / T_{2^{\prime}}
$$

In terms of the Carnot temperature $T$ and factor $\Phi$ the efficiency $\eta$, eq. (4), assumes the simple, pseudo-Carnot form

$$
\eta=1-\Phi \frac{T_{2}}{T^{\prime}} \text {. }
$$

The efficiency decrease is caused by the case of finite flows. The associated power of entropy production per unit time follows from Eqs. (5) and (6)

$$
\sigma_{s}=q_{1}\left(\frac{(\Phi-1)}{T^{\prime}}+\left(\frac{1}{T^{\prime}}-\frac{1}{T_{1}}\right)\right)
$$

or

$$
\sigma_{s}=\frac{T_{1^{\prime}}}{T^{\prime}} \sigma_{s}^{\mathrm{int}}+q_{1}\left(\frac{1}{T^{\prime}}-\frac{1}{T_{1}}\right) .
$$

The first of these equations can be applied immediately; the second calls for a function $T_{1},\left(T_{1}, q_{1}\right)$ as in eq. (12). When the upper heat exchange rate in both reservoirs depends on the difference of temperatures in the power $a \quad(a=4$ for radiative energy exchange and 1 for Newtonian one) i.e. for

$$
q_{1}=g_{1}\left(T_{1}^{a}-T_{1^{\prime}}^{a}\right)
$$

then, since $T_{1^{\prime}}=\left(T_{1}^{a}-q_{1} / g_{1}\right)^{1 / a}$, the following formula holds for the power of entropy generation

$$
\sigma_{s}=\frac{\left(T_{1}^{a}-q_{1} / g_{1}\right)^{1 / a}}{T^{\prime}} \sigma_{s}^{\mathrm{int}}+q_{1}\left(\frac{1}{T^{\prime}}-\frac{1}{T_{1}}\right) .
$$

This means that only in the "endoreversible" case, i.e. when the internal entropy production vanishes, the external entropy production is simply related to the product of heat $\mathrm{q}_{1}$ and the suitable difference of temperature reciprocals, $\left(T^{\prime}\right)^{-1}-\left(T_{1}\right)^{-1}$, as in the two-body contact. In the general case of a finite internal entropy production the external part of $\sigma_{\mathrm{s}}$ follows in terms of its internal part in the form

$$
\sigma_{s}^{e x t}=\left(\frac{\left(T_{1}^{a}-q_{1} / g_{1}\right)^{1 / a}-T^{\prime}}{T^{\prime}}\right) \sigma_{s}^{\text {int }}+q_{1}\left(\frac{1}{T^{\prime}}-\frac{1}{T_{1}}\right)
$$

so that the sum of both parts of the entropy production agrees with the formula (12).

Definition (7) may also be derived from the invariance of the entropy production with respect to variables transformation when passing from constrained traditional controls $T_{1}$, and $T_{2}$. to the unconstrained control $T$, We conclude that the analytical description of thermal energy converters in terms of the Carnot temperature is particularly simple.

The efficiency worsening caused by the dissipation is described in a general way by the transformed formula (1)

$$
\eta=\eta_{C}-T_{2} \sigma_{s} / q_{1}
$$

Of course the pseudo-Carnot formula, eq. (8), also belongs to the class of imperfect efficiencies of the type (14) as it can be given the form

$$
\eta=\eta_{C}-T_{2}\left(\frac{\Phi}{T^{\prime}}-\frac{1}{T_{1}}\right)
$$

which implies the ratio $\sigma_{s} / \mathrm{q}_{1}$ consistent with eq.(5) and (9). Various equations that describe the entropy production $\sigma_{\mathrm{s}}$, presented above, are helpful in definite situations when one wants to evaluate the efficiency worsening. Yet the knowledge of the entropy production $\sigma_{\mathrm{s}}$ is also necessary in calculations of generalized exergies considered in the final section of this paper. But in the dynamical cases essential is also the best time behavior of $\sigma_{\mathrm{s}}$.

\section{Heat Flux and Power In Steady Operations}

Consider steady thermal machines driven by fluids exhibiting nonlinear properties.

We begin with the symmetric nonlinear case in which the heat transfer rate is proportional to the difference of absolute temperatures in certain power $a$. The case of $a=4$ refers to the radiation, $a=-1$ to the Onsagerian kinetics and $a=1$ to the Fourier law of heat exchange. (In the Onsagerian case the quantities $g_{\mathrm{i}}$ are negative in the common formalism considered.)

Next we consider the "hybrid nonlinear case" in which the upper-temperature fluid is still governed by the kinetics proportional to the difference in $T^{\mathrm{n}}$ whereas the kinetics in the lower reservoir is Newtonian.

Here we consider symmetric nonlinear case. We assume that the energy exchange process in the upper reservoir satisfies equation (11), and that an equation of the same type and with the same coefficient $a$ is valid for the energy exchange in the lower reservoir, namely

$$
q_{2}=g_{2}\left(T_{2^{\prime}}^{a}-T_{2}^{a}\right)
$$

To express the internal balance equation for the entropy

$$
\Phi g_{1}\left(T_{1}^{a}-T_{1^{\prime}}^{a}\right) / T_{1^{\prime}}=g_{2}\left(T_{2^{\prime}}^{a}-T_{2}^{a}\right) / T_{2^{\prime}}
$$


in terms of $T^{\prime}$ and $T_{1}$, we substitute $T_{2^{\prime}} \equiv T_{1} T_{2} / T^{\prime}$ into (17). Next we solve the result obtained with respect to $T_{1}$. This leads to an equation describing (in terms of $T^{\prime}$ ) the upper temperature of the circulating fluid $T_{1}$,

$$
T_{1^{\prime}}=\left(T_{1}^{a}-g_{2} \frac{T_{1}^{a}-T^{\prime a}}{\Phi g_{1}\left(T^{\prime} / T_{2}\right)^{a-1}+g_{2}}\right)^{1 / a} .
$$

From this expression and equation (11) the heat flux $q_{1}$ follows in terms of $T$. This heat flux is obtained in the form

$$
q_{1}=g_{1} g_{2} \frac{T_{1}^{a}-T^{\prime a}}{\Phi g_{1}\left(T^{\prime} / T_{2}\right)^{a-1}+g_{2}}
$$

which represents "thermal characteristics" of the system. An expression for $T_{2}$, corresponding with (18) follows from the thermodynamic definition of Carnot temperature, $T_{2^{\prime}} \equiv T_{1} T_{2} / T^{\prime}$. Also, $q_{2}=q_{1}(1-\eta)$, where $\eta$ is defined by the pseudo-Carnot expression (8). Thus all necessary quantities are found. We observe that for $a=1$ the kinetics of heat exchange depends on the difference of two temperatures $T_{1}-T$, as in the case of direct two-body contact. Yet, in nonlinear processes the heat flux (19) emerges as function of three (not merely two) temperatures, $T, T_{1}$ and $T_{2}$. This means that the rule of the twobody contact (satisfied when $a=1$ ) is invalid in the case of nonlinear processes. Still we can evaluate associated power limits by maximizing power $p$ related to equation (19) with respect to the free Carnot control, $T$; ; see equation (22) below.

For $a=4$ the model describes the radiation engine usually called the Stefan-Boltzmann engine. In spite of the model's simplicity, its two "resistive parts" take rigorously into account the entropy generation caused by simultaneous emission and absorption of black-body radiation, the model's property which some of FTT adversaries seem not to be aware of. This entropy generation is just the external part of the total entropy production that follows as the "classical" sum:

$$
\sigma_{s}^{e x t}=q_{1}\left(T_{1^{\prime}}^{-1}-T_{1}^{-1}\right)+q_{2}\left(T_{2}^{-1}-T_{2^{\prime}}^{-1}\right),
$$

where each $\mathrm{q}_{\mathrm{i}}$ is given by the Stefan-Boltzmann-like law. For the "symmetric"kinetics governed by the differences in $T^{\mathrm{a}}$, the $T$ '-representation of the total entropy production in the system follows from equations (9) and (19)

$$
\sigma_{s}=g_{1} g_{2} \frac{T_{1}^{a}-T^{\prime a}}{\Phi g_{1}\left(T^{\prime} / T_{2}\right)^{a-1}+g_{2}}\left(\frac{(\Phi-1)}{T^{\prime}}+\left(\frac{1}{T^{\prime}}-\frac{1}{T_{1}}\right)\right) \text {. }
$$

The superiority of Carnot control $T$ ' over the heat flux control $q_{1}$ may be noted here. Since the heat-flux expression (19) cannot generally be inverted to get an explicit function $T^{\prime}\left(q_{1}\right)$, the analytical expression for the heat-flux representation of the entropy production or associated mechanical power $p$ cannot generally be found in an analytical form. Still we can express the entropy production and related power $p$ in terms of Carnot control, $T$, and then evaluate a limiting power by maximizing $p$ with respect to the free Carnot control, $T$. The appropriate power expression is

$$
p=q_{1} \eta=g_{1} g_{2} \frac{T_{1}^{a}-T^{\prime a}}{\Phi g_{1}\left(T^{\prime} / T_{2}\right)^{a-1}+g_{2}}\left(1-\Phi \frac{T_{2}}{T^{\prime}}\right) .
$$

In the case of analytical difficulties that may occur for $a$ different from the unity the maximization can easily be performed graphically by making the chart of $p(T)$ at all remaining variables kept constant. Thus, in general, the maximization can be performed analytically or graphically using the Carnot $T$, as the free control.

We consider now hybrid nonlinear case or the case when the radiation law governs the heat flow only in the upper reservoir, whereas it is the Newtonian model that governs the lower one

$$
q_{2}=g_{2}\left(T_{2^{\prime}}-T_{2}\right) \text {. }
$$

Again, the efficiency of the imperfect unit is satisfied by expression $\eta=1-\Phi T_{2}, / T_{1}$, To express the internal balance equation for the entropy

$$
\Phi g_{1}\left(T_{1}^{4}-T_{1^{\prime}}^{4}\right) / T_{1^{\prime}}=g_{2}\left(T_{2^{\prime}}-T_{2}\right) / T_{2^{\prime}}
$$

in terms of $T^{\prime}$ and $T_{1}$, we substitute $T_{2^{\prime}} \equiv T_{1^{\prime}} T_{2} / T^{\prime}$ into (24). This leads to $T$ ' in terms of $T_{1}$,

$$
\Phi g_{1}\left(T_{1}^{4}-T_{1^{\prime}}^{4}\right)=g_{2}\left(T_{1^{\prime}}-T^{\prime}\right)
$$

and then to the mechanical power $p$ in terms of $T_{1}$. The thermal efficiency of the engine can be obtained in the form using the temperature $T_{1}$, as an effective control variable

$$
\eta=1-\Phi \frac{T_{2}}{T^{\prime}}=1-\frac{\Phi T_{2}}{T_{1^{\prime}}-\Phi g_{1}\left(T_{1}^{4}-T_{1^{\prime}}^{4}\right) / g_{2}}
$$

This leads to the mechanical power expression with the explicit control $T_{1}$,

$$
p=q_{1} \eta=g_{1}\left(T_{1}^{4}-T_{1^{\prime}}^{4}\right)\left(1-\frac{\Phi T_{2}}{T_{1^{\prime}}-\Phi g_{1}\left(T_{1}^{4}-T_{1^{\prime}}^{4}\right) / g_{2}}\right)
$$

Since from Eq. (11) $T_{1^{\prime}}=\left(T_{1}^{a}-q_{1} / g_{1}\right)^{1 / a}$, the heat flux representation of the above equation is obtained in the form

$$
p=q_{1} \eta=q_{1}\left(1-\frac{\Phi T_{2}}{\left(T_{1}^{a}-q_{1} / g_{1}\right)^{1 / a}-\Phi q_{1} / g_{2}}\right) \text {. }
$$

Equations (27) or (28) allow analytical or graphical maximization of power with respect to a single control variable, $T_{1}$,or $\mathrm{q}_{1}$. This leads to the steady limits on power production in imperfect units. 
We note that a suitable control may be the Carnot temperature itself or a function or an operator of the remaining variables. The operator structure of $T$, is frequent in dynamical problems.

\section{Dynamical Setting for Finite Resources}

When resources become finite and/or the propelling fluid flows at a finite rate the driving temperature and other intense parameters decrease along the process path. The above analysis is generalized to take into account the decay of the thermal potential of resource in time or space. This means that the previous (steady) analysis need to be replaced by a dynamic one and the mathematical formalism transferred from the realm of functions to the realm of functionals. Here the optimization task is to find an optimal profile of the driving temperature $T$, along the resource path (fluid's path) that assures the minimum of the integral entropy production and - simultaneously - the extremum of the work consumed or delivered (Fig.2).

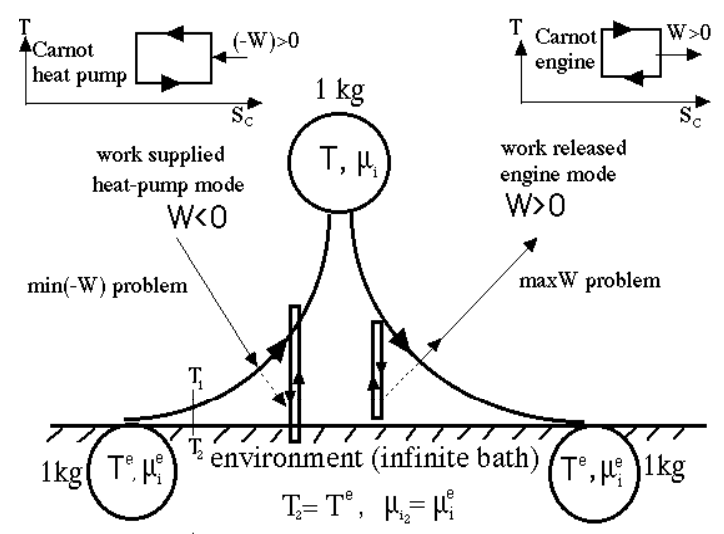

Fig. 2. The dynamic work limit found for a system of a resource and infinite bath leads to a generalized exergy evaluated for a finite duration of the state change and a minimal irreversibility.

In dynamical systems differential forms of expressions are necessary. For a suitably defined time variable $\tau$ (associated with the resource fluid; see eq. (32) below) and an arbitrary type of heat transfer (Newtonian or not) the internal entropy production is

$$
S_{\sigma}^{\text {int }}=-\int_{\tau^{i}}^{\tau^{f}} c(T) \frac{\Phi-1}{T^{\prime}\left(T_{1}, \dot{T}\right)} \dot{T}_{1} d \tau_{1}
$$

whereas its external part

$$
S_{\sigma}^{e x t}=-\int_{\tau^{i}}^{\tau^{f}} c(T)\left(\frac{1}{T^{\prime}\left(T_{1}, \dot{T}_{1}\right)}-\frac{1}{T_{1}}\right) \dot{T}_{1} d \tau_{1}
$$

The total entropy production, which determines the lost work in equations of extended availabilities is the integral

$$
S_{\sigma}=-\int_{\tau^{i}}^{\tau^{f}} c(T)\left(\frac{\Phi}{T^{\prime}}-\frac{1}{T_{1}}\right) \dot{T_{1}} d \tau_{1}
$$

The limiting production or consumption of mechanical energy is associated with extremum power or the minimal sum of functionals (29) and (30) or minimum of the overall entropy production (31). These equations assume that it is possible to determine explicit form of functions describing the Carnot temperature $T$ ' in terms of the current fluid's temperature $T$ and its time derivative. Such functional structure would allow to apply the variational calculus in the optimization analysis. If this function is impossible to find in an explicit form then equations (29) and (30) should be written in the form in which $T$ and $T_{1}$ are two separable variables in the Pontryagin's algorithm of the optimal control. In this case a differential constraint must be added that limits the changes $\mathrm{d} T_{1} / \mathrm{d} t$ with the state variable $T_{1}$ and control $T$ '(see eq. (33) below).

We shall now specialize to what we called the symmetric nonlinear case. It involves the radiative heat transfer $(a=4)$ in both upper and lower reservoirs and corresponds with the form (22) of the intensity of total entropy production.

We can define the nondimensional time $\tau_{1}$ by the equality

$$
q_{1} / g_{1}=-G_{1} c\left(T_{1}\right) d T_{1} /\left(\alpha_{1} a_{v} F_{1} d x\right) \equiv-d T_{1} / d \tau_{1}
$$

which means that the driving heat flux can be measured in terms of the temperature drop of the propelling fluid per unit of the nondimensional time. Comparing the result obtained with $q_{1}$ of equation (20) we obtain the basic differential equation

$$
d T_{1} / d \tau_{1}=-g_{2} \frac{T_{1}^{a}-T^{\prime a}}{\Phi g_{1}\left(T^{\prime} / T_{2}\right)^{a-1}+g_{2}}
$$

which is the differential constraint in the problem of minimization of the total entropy production (31) by Pontryagin's maximum principle. However, it should be noted that the method of variational calculus cannot effectively be applied (see the related argument below).

We shall now specialize to what we called the hybrid nonlinear case. It involves the radiative heat transfer $(a=4)$ in the upper reservoir and a convective one in the lower one $\left(\dot{T}_{1} \equiv d T_{1} / d \tau_{1}\right)$

$$
S_{\sigma}^{\mathrm{int}}=-\int_{\tau^{i}}^{\tau^{f}} c\left(T_{1}\right) \frac{\Phi-1}{\left(T_{1}^{a}+\dot{T}_{1}^{a}\right)^{\frac{1}{a}}+\dot{T}_{1} \Phi g_{1} / g_{2}} \dot{T}_{1} d \tau_{1}
$$

and

$$
S_{\sigma}^{e x t}=-\int_{\tau^{i}}^{\tau^{f}} c\left(T_{1}\right)\left(\frac{1}{\left(T_{1}^{a}+\dot{T}_{1}^{a}\right)^{\frac{1}{a}}+\dot{T}_{1} \Phi g_{1} / g_{2}}-\frac{1}{T_{1}}\right) \dot{T}_{1} d \tau_{1}
$$

To obtain an optimal path associated with the limiting production or consumption of mechanical energy the sum of the above functionals i.e the overall entropy production 


$$
S_{\sigma}=-\int_{\tau^{i}}^{\tau^{f}} c\left(T_{1}\right)\left(\frac{\Phi}{\left(T_{1}^{a}+\dot{T}_{1}^{a}\right)^{\frac{1}{a}}+\dot{T}_{1} \Phi g_{1} / g_{2}}-\frac{1}{T_{1}}\right) \dot{T}_{1} d \tau_{1}
$$

has to be minimized for a fixed duration and defined end states of the fluid. The most typical way to do accomplish the minimization is to write down and then solve the EulerLagrange equation of the variational problem. Analytical solutions are seldom, however, thus one has to rest on numerical techniques.

\section{Thermodynamic Aspects of Nonliner Kinetics}

In general equations of nonlinear macrokinetics a recent approach considers coupled transfer of mass $(\mathrm{m})$ and heat $(\mathrm{h})$. Introduced are potentials $F_{\mathrm{i}}=\left(1 / T,-\mu_{\mathrm{i}} / T\right), \mathrm{i}=0,1 \ldots n$, which are the thermodynamic conjugates of the extensive variables in the Gibbs equation for the system's entropy

$$
d S=T^{-1} d E-T^{-1} \mu_{\alpha} d c_{\alpha} \equiv \sum_{i=0}^{s} F_{i} d C_{i} \equiv \mathbf{F} . d \mathbf{C}
$$

The process kinetics is described by the general exchange equation for the net flux $J_{\mathrm{i}}$

$$
J_{i}=I_{j}^{0}\left\{\exp \left(-\sum_{i} v_{i k}^{*} F_{k}^{f} / \mathrm{R}\right)-\exp \left(-\sum_{i} v_{i k}^{*} F_{k}^{b} / \mathrm{R}\right)\right\}
$$

whose equivalent form in terms of deviations from equilibrium is

$$
J_{i}=I_{i}^{e q} \Delta \exp \left(-\sum_{i} v_{i k}^{*}\left(F_{k}^{f}-F_{k}^{e q}\right) / \mathrm{R}\right)
$$

with

$$
I_{i}^{e q} \equiv I_{i}^{0} \exp \left(-\sum v_{i k}^{*} F_{k}^{e q} / \mathrm{R}\right)
$$

as the common value of the absolute current at equilibrium.

In the case of pure heat exchange the corresponding kinetic set contains just one equation that describes the nonlinear heat flux. The corresponding kinetic set describing the contains two equations. The first one describes the heat flux driving the engine

$$
q_{1}=I_{1}^{e q} \Delta \exp \left(-E\left(T^{-1}-T^{-1^{e q}}\right) / \mathrm{R}\right)
$$

where

$$
I_{1}^{e q} \equiv I_{1}^{0} \exp \left(-E T^{-1^{e q}} / \mathrm{R}\right)
$$

The constant $E$ is an activation energy. Whence, close to equilibrium, a linear approximation of the nonlinear exchange equation follows as

$$
q_{1}=I_{1}^{e q} E \mathrm{R}^{-1}\left(T^{-1^{\prime}}-T^{-1}\right) \cong I_{1}^{e q} E \mathrm{R}^{-1}\left(T-T_{1^{\prime}}\right) / T T_{1^{\prime}}
$$

Comparing this expression with the classical Newton's structure $q_{1}=g_{1}\left(T_{1}-T_{1^{\prime}}\right)$ we find that the conductance in the present model varies with $T$ in accordance with the equation

$$
g_{1}=I_{1}^{e q} E \mathrm{R}^{-1} / T T_{1^{\prime}}=I_{1}^{0} \exp \left(-E T^{1^{e q}} / \mathrm{R}\right) E \mathrm{R}^{-1} / T T_{1^{\prime}}
$$

This shows that the correspondence with classical equation of heat exchange can be assured. Above equation may serve to estimate the value of the exchange current provided that the heat exchange coefficient $a$, constant $E$ and an equilibrium or average temperature of two bodies is known. It should be realized that $E$ characterizes the temperature dependence of heat conductivity. We can now write down the kinetic equations for both reservoirs.

For the known process coefficients the heat exchange equation for the first reservoir has the form

$$
q_{1}=I_{1}^{0}\left\{\exp \left(-E_{1} / \mathrm{R} T_{1}\right)-\exp \left(-E_{1} / \mathrm{R} T_{1^{\prime}}\right)\right\}
$$

whereas that for the second fluid is

$$
q_{2}=I_{2}^{0}\left\{\exp \left(-E_{2} / \mathrm{R} T_{2^{\prime}}\right)-\exp \left(-E_{2} / \mathrm{R} T_{2}\right)\right\}
$$

On this ground one can develop the nonlinear theory in which thermal conductivities and related conductances are variable i.e. are state functions.

\section{Newtonian Fluids}

Analytical solutions are possible for linear problems in particular. For the Newtonian heat exchange in both reservoirs $(a=1)$ the above formulae simplify to the forms

$$
S_{\sigma}^{\mathrm{int}}=-\int_{\tau^{i}}^{\tau^{f}} c \frac{\Phi-1}{T_{1}+\dot{T}_{1}\left(1+\frac{g_{1}}{g_{2}} \Phi\right)} \dot{T}_{1} d \tau_{1}
$$

and

$$
S_{\sigma}^{e x t}=-\int_{\tau^{i}}^{\tau^{f}} c\left(\frac{1}{T_{1}+\dot{T}_{1}\left(1+\frac{g_{1}}{g_{2}} \Phi\right)}-\frac{1}{T_{1}}\right) \dot{T}_{1} d \tau_{1} .
$$

Their sum constitutes the functional of the total entropy production that is the basics for the linear theory developed earlier [13]. Still the linear theory presented here is more general than the cited previous theory since the latter is here generalized to the imperfect energy generators (consumers) associated with $\Phi$ different than the unity.

Equations (34)-(36) contain expressions representing the Carnot temperature $T$ in terms of the upper reservoir temperature $T_{1}$ and the time derivative of this quantity. In fact, these equations prove that the success in achieving Lagrange 
functionals (necessary when one wants to apply the classical method of calculus of variations) is crucially dependent on the possibility of getting Carnot temperature $T$, in the form of an explicit analytical function of $T$, and $\mathrm{d} T / \mathrm{d} t$. In the case of the symmetric nonlinear model (radiative exchange on both sides of the engine) such explicit function was impossible to find, yet as we have shown now the possibility exists in the case of the hybrid nonlinear model. For the latter model one can therefore write down explicit Euler-Lagrange equations of the variational problem and perform extremization of functionals describing either the entropy production or produced (consumed) power. In fact, our earlier work [13] shows that such functionals yield the same optimal trajectory whenever fixed end states are assumed at both ends of the process.

For a Newtonian fluid the minimization of the entropy production integral of the dynamical problem involves the extension of the established endoreversible formula [13] to the case taking the internal dissipation into account

$$
S_{\sigma}=c \int_{\tau^{i}}^{\tau^{f}}\left(\frac{\dot{T}}{T(T+\dot{T})}-\frac{\Phi-1}{T+\dot{T}}\right) \dot{T} d \tau .
$$

The optimal solution can be obtained by the variational calculus; it proves that an unconstrained extremal is an exponential curve satisfying the optimality condition

$$
\frac{d T}{d \tau}=\xi(\Phi) T
$$

The modified non-dimensional time $\tau$, or the ratio of pipeline length $x$ and the height of the transfer unit $H T U$ related to overall $g$ ' of Eq. (10), is identical with the ( $\Phi$ dependent) overall number of transfer units. $\xi(\Phi)$ is the rate indicator which is positive for the fluid's heating and negative for fluid's cooling. From the problem boundary conditions of the problem the numerical value of the $\xi$ follows.

As it follows form the definition of $\Phi$ in equation (4)

$$
\sigma_{s}^{\text {int }}=T_{1^{\prime}}^{-1} q_{1}(\Phi-1)
$$

This equation can be used to prove that the second term in (49) does not represent the sole effect of the internal dissipation. The same conclusion holds for the general nonlinear model. For this model we find

$$
\sigma_{s}^{\text {int }} / q_{1}=\frac{(\Phi-1)}{\left(T_{1}^{a}-g_{2} \frac{T_{1}^{a}-T^{\prime a}}{\Phi g_{1}\left(T^{\prime} / T_{2}\right)^{a-1}+g_{2}}\right)^{1 / a}}
$$

and

$$
\begin{aligned}
& \sigma_{s}^{e x t} / q_{1}=\left(\frac{1}{T^{\prime}}-\frac{1}{T_{1}}\right)+ \\
& +(\Phi-1)\left(\frac{1}{T^{\prime}}-\frac{1}{\left(T_{1}^{a}-g_{2} \frac{T_{1}^{a}-T^{\prime a}}{\Phi g_{1}\left(T^{\prime} / T_{2}\right)^{a-1}+g_{2}}\right)^{1 / a}}\right)
\end{aligned}
$$

In fact, each of two additive parts of functional (49) or the additive parts of nonlinear functionals discussed can contribute to the external and internal dissipation.

\section{Generalized Exergy as a Dynamic Limit}

Carnot temperature control ensuring the extremum of work associated with the functional (49) is

$$
\begin{aligned}
& T^{\prime}(\tau)=T(\tau)(1+\xi(\Phi)) \\
& =T^{i}\left(T^{f} / T^{i}\right)^{\left(\tau-\tau^{i}\right) /\left(\tau^{f}-\tau^{i}\right)}\left(1+\ln \left(T^{f} / T^{i}\right) /\left(\tau^{f}-\tau^{i}\right)\right)
\end{aligned}
$$

It corresponds with the power expression (28) in the case $a=1$. Its integration along the optimal path with respect to time leads the generalized availability

$$
A^{\infty}=A^{\text {class }}+c(1-\Phi) T^{e} \ln \left(T / T^{e}\right) \pm c T^{e} \Phi \frac{\left[\ln \left(T / T^{e}\right)\right]^{2}}{\tau^{f}-\tau^{i} \pm \ln \left(T / T^{e}\right)}
$$

The properties of this function may be depicted in a draft that illustrates the effect of internal irreversibilities $\Phi$ on limiting finite-rate work yield in engines and consumed in heat pumps.

Generalized exergy of limiting continuous process, $A^{\infty}$, prohibits processes from operating below the heat-pump mode (the lower bound for work supplied) and above the engine mode line (the upper bound for work produced). The socalled endoreversible limits correspond with $\Phi=1$; weaker limits of classical exergy are represented by the straight line $A=A_{\text {class }}$. Regions of possible improvements are found when imperfect machines are replaced by those with better performance, including limits for Carnot machines.

The classical thermal availability contained in this equation is defined in the standard way

$$
A^{\text {class }} \equiv c\left(T-T^{e}\right)-c T^{e} \ln \left(T / T^{e}\right) .
$$

The classical availability is the potential or the state function whose change between two arbitrary states describes the reversible work. On the other hand, generalized availability functions are irreversible extensions of this classical function including minimally irreversible processes. Note that the mean process efficiency or the ratio $A^{\infty} / Q_{1}$ is lower than the pseudo-Carnot efficiency (8) due to the finiteness of the resource flow and the corresponding decrease of the resource temperature as the process advances in time.

\section{Conclusion}

The obtained exergy functions are discrete generalizations of the continuous dissipative exergy for the case of imperfect stages. The generalized exergy in processes departing from the equilibrium (upper sign) is larger than the one in processes approaching the equilibrium (lower sign). This is because one respectively adds or subtracts the product of $T^{\mathrm{e}}$ and entropy 
production in equations describing the generalized availability. We observe that the limits for mechanical energy yield or consumption provided by exergies $A^{\infty}$ are always stronger than those defined by the classical exergy. Thus, in both modes the generalized exergies provide enhanced bounds in comparison with those predicted by classical exergy. Equation (42) shows that both internal and external dissipation increase the minimum work that must be supplied to the system. Likewise, both sort of dissipations decreases the maximum work that can be produced by the system.

Therefore functions $A^{\infty}$ provide work limits which take into account limitations resulting from finite rates of external transports and internal irreversibilities. The use of concepts developed to systems with mass transfer and chemical reactions is the subject of the current effort.

\section{Acknowledgments}

This work was supported by the Polish Committee of National Research (KBN), grant T09C 02426.

\section{Nomenclature}

$A^{\infty}$ generalized exergy of a continuous process

$A^{\text {class }}$ classical available energy (exergy)

$C$ specific heat at the constant pressure

$G$ molar mass flux, total flow rate

$g_{1}, g$ partial and overall conductances

$H_{T U}$ height of transfer unit

$P, p$ cumulative power output and power output at a stage

$\mathrm{Q}_{1}$ cumulative heat

$q_{1}$ driving heat power

$R(x, t)$ optimal work of cost type in terms of state and time

$r_{l}, r_{2}$ resistances, reciprocals of conductances $g_{l}$ and $g_{2}$

$S$ entropy of controlled phase

$\Delta S_{1}$, entropy change of circulating fluid along isotherm $T_{1}$,

$\Delta S_{2}$, entropy change of the circulating fluid along sotherm $\mathrm{T}_{2}$,

$S_{\sigma}$ specific entropy production

$T$ temperature of controlled phase

$T_{1}, T_{2}$ bulk temperatures of fluids 1 and 2

$T_{1}, T_{2}$ temperatures of circulating fluid (Fig. 1)

$T^{e}$ constant equilibrium temperature of environment

$T^{\prime}$ Carnot temperature, temperature of controlling phase

$t$ physical time, contact time

$\dot{T}=d T / d \tau$ rate of temperature change as control variable

$V=\max W$ optimal work function of profit type

$W=P / G$ total specific work or total power per unit mass flux

$x$ transfer area coordinate

\section{Greek Symbols}

$\alpha$ 'overall heat transfer coefficient

$\eta=\mathrm{p} / \mathrm{q}_{1}$ first-law efficiency

$\Phi$ factor of internal irreversibility $\sigma_{\mathrm{s}}$ cumulative entropy production

tnondimensional time, number of the heat transfer units

$\left(x / H_{\mathrm{TU}}\right)$

Subscripts

$i$ i-th state variable

mpmaximum power point

1, 2first and second fluid

Superscrits

eenvironment, equilibrium

ffinal state

iinitial state

$N$ total number of stages

\section{References}

[1] T.J. Kotas, Exergy Method of Thermal Plant Analysis, Butterworths, Borough Green (1985), pp. 2-19.

[2] R.E. Bellman, Adaptive Control Processes: a Guided Tour, Princeton Univ. Press, Princeton (1961), pp. 1-35.

[3] L.T. Fan, The Continuous Maximum Principle, A Study of Complex System Optimization, Wiley, New York (1966), pp. 329-342.

[4] S. Sieniutycz, Optimization in Process Engineering, 2nd e., Wydawnictwa Naukowo Techniczne, Warszawa (1991), pp. 151-194.

[5] H. Rund, The Hamilton-Jacobi Theory in the Calculus of Variations, Van Nostrand, London (1966), pp. 1-32.

[6] L.S. Pontryagin, V.A. Boltyanski, R. Gamkrelidze, and E.F. Mischenko, The Mathematical Theory of the Optimal Processes, Wiley, New York (1962), pp. 13-85.

[7] R. Aris, Discrete Dynamic Programming, Blaisdell, New York (1964), pp. 10-39.

[8] R.S. Berry, V.A. Kazakov, S. Sieniutycz, Z. Szwast, and A.M. Tsirlin, Thermodynamic Optimization of Finite Time Processes, Wiley, Chichester (2000), p.117.

[9] F.L.Curzon and B. Ahlborn, B., "Efficiency of Carnot engine at maximum power output", Am. J. Phys. (1975), Vol. 43, pp. 22-24.

[10] M.J. Ondrechen, B. Andresen, M. Mozurkiewicz, and R.S. Berry, "Maximum Work from a Finite Reservoir by Sequential Carnot Cycles", Am. J. Phys. (1981) Vol. 49, 681-685.

[11] A. Bejan and M.R. Errera, "Maximum Power from a Hot Stream”, Int. J. Heat Mass Transfer, (1998) Vol. 41, pp. 2025-2036.

[12] S. Sieniutycz and Z. Szwast, "Work Limits in Imperfect Sequential Systems with Heat \& Fluid Flow", $J$. Nonequilib. Thermodyn., (2003) Vol. 28, pp.85-114.

[13] S. Sieniutycz, "Nonlinear Thermokinetics of Maximum Work in Finite Time", Int. J. Engng Sci., (1988) Vol. 36, pp. 557-597. 